\title{
Rapid and Simplified Induction of Neural Stem/Progenitor Cells (NSCs/NPCs) and Neurons from Human Induced Pluripotent Stem Cells (hiPSCs) Ryutaro Kajihara ${ }^{1,2,3}$, Tadahiro Numakawa ${ }^{1, *}$ and Takumi Era ${ }^{1}$
}

\author{
1Department of Cell Modulation, Institute of Molecular Embryology and Genetics, Kumamoto University, \\ Kumamoto, Japan; 'Department of Biomedical Laboratory Sciences, Faculty of Life Sciences, \\ Kumamoto University, Kumamoto, Japan; ${ }^{3}$ Roswell Park Comprehensive Cancer Center, Center for \\ Immunotherapy, Buffalo, NY, USA \\ *For correspondence: numakawa.yyrmk@gmail.com
}

[Abstract] Human induced pluripotent stem cells (iPSCs) and their progeny displaying tissue-specific characteristics have paved the way for regenerative medicine and research in various fields such as the elucidation of the pathological mechanism of diseases and the discovery of drug candidates. iPSCderived neurons are particularly valuable as it is difficult to analyze neural cells obtained from the central nervous system in humans. For neuronal induction with iPSCs, one of the commonly used approaches is the isolation and expansion of neural rosettes, following the formation of embryonic bodies (EBs). However, this process is laborious, inefficient, and requires further purification of the cells. To overcome these limitations, we have developed an efficient neural induction method that allows for the generation of neural stem/progenitor cells (NSCs/NPCs) from iPSCs within 7 days and of functional mature neurons. Our method yields a PAX6-positive homogeneous cell population, a cortical NSCs/NPCs, and the resultant NSCs/NPCs can be cryopreserved, expanded, and differentiated into functional mature neurons. Moreover, our protocol will be less expensive than other methods since the protocol requires fewer neural supplements during neural induction. This article also presents the FM1-43 imaging assay, which is useful for the presynaptic assessment of the iPSCs-derived human neurons. This protocol provides a quick and simplified way to generate NSCs/NPCs and neurons, enabling researchers to establish in vitro cellular models to study brain disease pathology.

Keywords: Human Induced Pluripotent Stem Cell, Neural Stem Cell, Neural Progenitor Cell, Neuron, Neural Induction, FM1-43

[Background] Human iPSCs were first established in 2007 through the reprogramming of dermal fibroblasts using four transcription factors (Oct4, Sox2, Klf4, and c-Myc) and exhibited similar characteristics of embryonic stem cells (ESCs), including their pluripotency and self-renewal (Takahashi et al., 2007; Yu et al., 2007). Since iPSCs overcome the limitation of the ethical problems and the allogenic immune rejection, which ESCs also have, they are highly expected to be applied to regenerative medicine. Importantly, researchers can establish disease-specific iPSCs from patients' somatic cells, including skin fibroblasts, keratinocytes, cord blood-derived endothelial cells, peripheral blood mononuclear cells, and surprisingly tumor cells (Raab et al., 2014; Marin Navarro et al., 2018; Saha et al., 2018). 
Driving disease iPSCs into tissue-specific cells enables us to obtain target cells, study the diseases' etiology and pathogenesis, and evaluate the efficacy or toxicity of drugs using the patients' cells (Inoue et al., 2014). Before establishing iPSC technology, it was almost impossible to analyze the affected neural cells in the central nervous system from patients with neurodegenerative diseases as the biopsy is not always available for the human brain. Therefore, the iPSC-based disease models are expected to be a powerful approach in elucidating the pathogenesis of neurodegenerative diseases and in drug discovery because they mimic pathological conditions that have not been easily reproduced in animal models and cultured cell lines (Dimos et al., 2008; Soga et al., 2015; Matsushita et al., 2019; Kajihara et al., 2020; Kido et al., 2020). So far, neuronal cells derived from disease-specific iPSCs in vitro have been used to study the pathology of neurodegenerative diseases including Alzheimer's disease, Parkinson's disease, and amyotrophic lateral sclerosis (ALS), and they accurately recapitulated the pathological changes that occur in the patients' brain (Dimos et al., 2008; Inoue et al., 2014).

The generation of disease-derived iPSCs was first established in 2008 by Dimos et al. using fibroblasts obtained from patients with familial ALS (Dimos et al., 2008). They induced ALS-specific iPSCs into motor neurons via EB formation. For many years, the major methods for neural induction from human ESCs and iPSCs have been either co-culture with a stromal cell line (Kawasaki et al., 2000; Lee et al., 2007) or the formation of EBs (Nemati et al., 2011; Falk et al., 2012; Shi et al., 2012), which involve the time-consuming steps, resulting in low-efficiency derivation of NSCs/NPCs. In the EB-based protocol, iPSCs are plated on a low adherence plate to form EBs, where EBs gradually aggregate over 5-7 days. These EBs are then transferred to a plate that supports cell adhesion, allowing the EBs to adhere to the bottom of the plate and spread in a neural rosette shape. From these rosettes, NSCs can be generated and passaged to form relatively stable NPCs. NPCs can then be plated in a neuron induction medium to be differentiated into mature neurons. Each of these methods (i.e., co-culture with a stromal cell line, or the formation of EBs) has contributed significantly to the field's advancement. However, some of these methods are not easily reproducible due to the variability of the iPSC lines or are not scalable to the extent needed for disease modeling, high-throughput screening, or cell therapy. Therefore, a simplified NSCs/NPCs generation protocol needs to be developed to the demands of research in brain development and neurological disorders.

Recent studies have shown that human iPSCs can be induced even into a monolayer neural culture in which iPSCs are plated on a defined matrix and exposed to growth factors (Chambers et al., 2009; Li et al., 2011; Yan et al., 2013). The monolayer-based protocols differ primarily in that iPSC colonies are maintained as a monolayer during neural induction and are differentiated directly into NSCs/NPCs without aggregation. Moreover, studies also demonstrated that dual SMAD inhibition enhances the efficiency of neural induction from iPSCs, in which small-compound inhibitors, SB431542 and dorsomorphin, block TGF $\beta /$ activin/nodal and BMP signaling, respectively (Chambers et al., 2009; Morizane et al., 2011). It is well-known that TGF $/$ activin/nodal signaling activates SMAD2/3 and promotes mesoderm induction (Dunn et al., 2004; Nishikawa et al., 2007; Era et al., 2008), while BMP signaling activates SMAD1/5/8 and induces mesendoderm differentiation (Tada et al., 2005; Wang and Chen, 2016). Thus, inhibiting these two SMAD signals results in promoting the ectoderm differentiation, 
including neural cells.

Herein, we describe a methodology for rapid and reproducible induction to the NSCs/NPCs from iPSCs within a week in a monolayer culture system without the process of EB formation. Our method yields a PAX6-positive homogeneous cell population, a cortical NSCs/NPCs (Matsushita et al., 2019; Kajihara et al., 2020). Moreover, differentiated mature neurons via the NSCs/NPCs by our protocol are capable of neurotransmission revealed by FM1-43 imaging assay.

\section{Materials and Reagents}

1. 100-mm dish (Falcon, catalog number: 353003 )

2. Glass pasteur pipette (Fisherbland, catalog number: 13-678-20C)

3. $200 \mu$ pipette tip (Greiner, catalog number: 739291)

4. 6-well plate (Falcon, catalog number: 353046 )

5. 15-ml conical tube (CORNING, catalog number: 430791)

6. $10 \mathrm{ml}$ pipette (VIOLAMO, catalog number: 2-5237-04)

7. Glass bottom dish (Matsunami Glass Ind., catalog number: D1113OH)

8. Cryovial (Nunc, catalog number: 340711 )

9. Geltrex hESC-Qualified, Ready-To-Use, Reduced Growth Factor Basement Membrane Matrix (Thermo Fisher Scientific, Gibco, catalog number: A1569601)

10. Collagenase Type IV (1 mg/ml) (STEMCELL Technologies, catalog number: 07909)

11. PBS without $\mathrm{CaCl}_{2}$ and $\mathrm{MgCl}_{2}$ (Thermo Fisher Scientific, Gibco, catalog number: 14190)

12. Accutase Cell Dissociation Reagent (Funakoshi, catalog number: AT104-500)

13. Trypan Blue Solution (Thermo Fisher Scientific, Gibco, catalog number: 15250061)

14. ROCK Inhibitor Y27632 (PeproTech, BioGems, catalog number: 1293823)

15. $\mathrm{NaCl}$ (WAKO, catalog number: 198-01675)

16. KCl (WAKO, catalog number: 163-03545)

17. $\mathrm{CaCl}_{2} \cdot 2 \mathrm{H}_{2} \mathrm{O}$ (WAKO, catalog number: 033-25035)

18. $\mathrm{MgCl}_{2} \cdot 6 \mathrm{H}_{2} \mathrm{O}$ (WAKO, catalog number: 135-00165)

19. $\mathrm{NaHCO}_{3}$ (WAKO, catalog number: 191-01305)

20. Glucose (WAKO, catalog number: 049-31165)

21. Polyethylenimine (WAKO, catalog number: 167-17811)

22. DMEM/F12 (SIGMA, catalog number: D8900-1L)

23. NON-ESSENTIAL AMINO ACID $(\times 100)$ (SIGMA, catalog number: M7145)

24. $200 \mathrm{mM}$ L-GLUTAMINE (Thermo Fisher Scientific, Gibco, catalog number: 25030-081)

25. KnockOut Serum Replacement (Thermo Fisher Scientific, Gibco, catalog number: 10828-028)

26. 2-Mercaptoethanol (Wako, catalog number: 131-14572)

27. bFGF (basic fibroblast growth factor) (Wako, catalog number: 060-04543)

28. BSA (bovine serum albumin) (Wako, catalog number: 012-23381) 
29. PSC Neural Induction Medium (consists of Neurobasal Medium and Neural Induction Supplement, 50×) (Thermo Fisher Scientific, Gibco, catalog number: A1647801)

30. Advanced DMEM/F12 (Thermo Fisher Scientific, Gibco, catalog number: 12634)

31. DMSO (dimethyl sulfoxide) (Sigma-Aldrich, catalog number: D2650)

32. B-27 Supplement (50×) (Thermo Fisher Scientific, Gibco, catalog number: 17504044)

33. Hoechst 33258 (Thermo Fisher Scientific, Invitrogen, catalog number: H3569)

34. Anti-SOX1 (Cell Signaling Technology, catalog number: 4194S)

35. Anti-NESTIN (R\&D systems, catalog number: MAB1259)

36. Anti-PAX6 (Covance, catalog number: PRB-278P)

37. Anti-Polysialic Acid (PSA)-NCAM (MilliporeSigma, catalog number: MAB5324)

38. Anti-Tuj1 (MilliporeSigma, catalog number: MAB1637)

39. Anti-MAP2 (MilliporeSigma, catalog number: Ab5622)

40. Alexa 488-conjugated donkey anti-rabbit IgG (Thermo Fisher Scientific, Invitrogen, catalog number: A21206)

41. Alexa 488-conjugated goat anti-mouse IgM (Thermo Fisher Scientific, Invitrogen, catalog number: A21042)

42. Alexa 594-conjugated donkey anti-rabbit IgG (Thermo Fisher Scientific, Invitrogen, catalog number: A21207)

43. Alexa 594-conjugated goat anti-mouse IgG (Thermo Fisher Scientific, Invitrogen, catalog number: A11005)

44. FM1-43 (Thermo Fisher Scientific, Invitrogen, catalog number: T3163)

45. 4\% Paraformaldehyde Phosphate Buffer Solution (WAKO, catalog number: 161-20141)

46. Triton X-100 (Nacalai, catalog number: 35501-15)

47. Double Neubauer Counting Chamber Set (NIPPON Genetics, catalog number: 15170-089)

48. iPSC medium (see Recipes)

49. Neural Induction Medium (see Recipes)

50. Neural Expansion Medium (see Recipes)

51. NSC freezing medium (see Recipes)

52. Polyethylenimine (PEI) solution (see Recipes)

53. Neuronal Medium (see Recipes)

54. Neuronal Maturation Medium (see Recipes)

55. Artificial Cerebrospinal Fluid (see Recipes)

\section{Equipment}

1. Biological safety cabinet (Dalton, model: DFC79)

2. Pipettes (Fisher, catalog number: 4680100)

3. Water bath (AS ONE, model: TR-3 $\alpha$ )

4. Inverted microscope (Olympus, model: CKX53) 
5. Incubator (Thermo Fisher, catalog number: 51030287)

6. Centrifuge (Kubota, model: 6200)

7. Milli-Q (MILLIPORE, model: C79625)

8. Fluorescence microscope (Leica, model: DMi8)

\section{Software}

1. Excel 2019 (Microsoft, https://www.microsoft.com/en-ca/microsoft-365/excel)

2. Leica Application Suite $X \quad($ LAS $X)$ (Leica, https://www.leicamicrosystems.com/products/microscope-software/p/leica-las-x-ls/)

\section{Procedure}

Figure 1 shows the schematic diagrams for neural differentiation using human iPSCs via NSCs/NPCs.

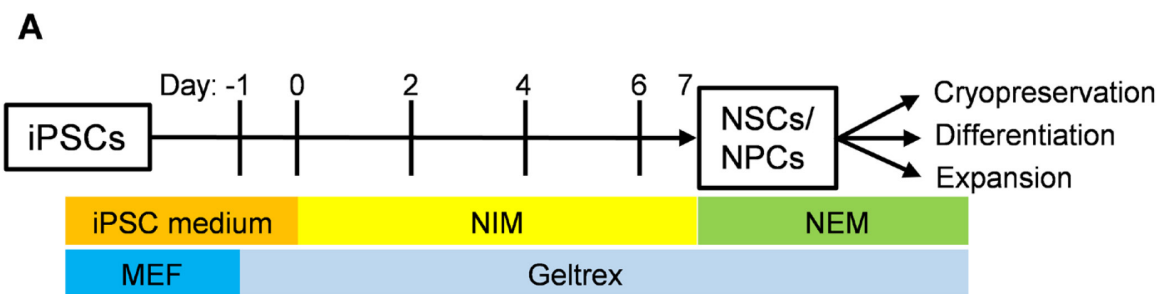

B

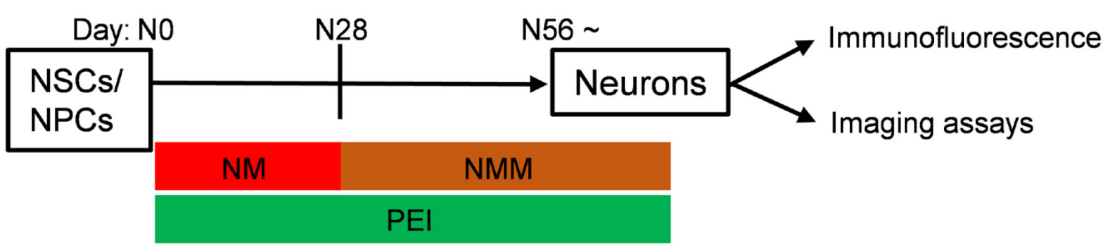

Figure 1. Schematic diagrams of neural induction (for NSCs/NPCs and neurons) from human iPSCs. Time points of differentiation, corresponding steps, and culture conditions are shown. A. NSCs/NPCs induction from iPSCs (days -1-7). B. Neuronal differentiation from NSCs/NPCs (neuronal day 0: NO-N56).

A. The preparation of human iPSCs for neural differentiation ( day -1$)$

1. Before using, disinfect the working area inside a biological safety cabinet by exposing it to UV light for $1 \mathrm{~h}$, and wipe out tools and supplies used in the cabinet with $70 \%$ ethanol.

2. Start with high-quality human iPSCs (with no differentiated colonies) grown on cell feeder of mitomycinC-treated MEF in iPSC medium (Recipe 1) on a 100-mm dish (Figure 2A). iPSCs are passaged every 3-7 days when they reach $70-80 \%$ confluence.

Notes: 
a. If you recognize differentiated colonies of iPSCs, remove the differentiated areas using a glass pasteur pipette or $200 \mu$ pipette tip under an inverted microscope kept in the biological safety cabinet.

b. The efficiency of successful neural induction depends on the quality of iPSCs. Therefore, we recommend that you examine several iPSC lines for neural differentiation.
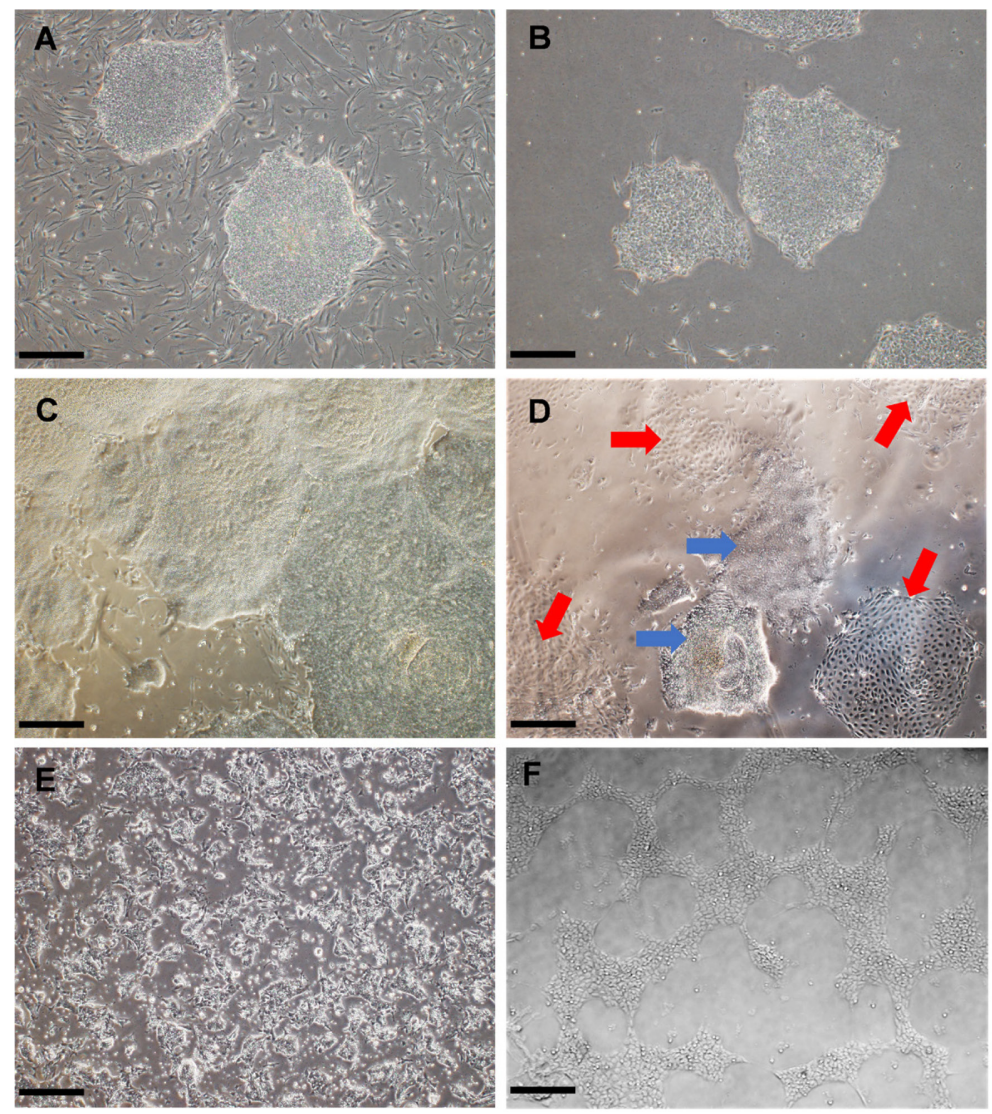

Figure 2. Representative phase-contrast images of cells during neural differentiation from iPSCs into NSCs/NPCs. A. Undifferentiated iPSC colonies kept on MEF feeder cells before starting neural induction. B. iPSC colonies on a Geltrex-coated well on the day of starting the induction. C. Day 7 of successful neural induction, PO NSCs/NPCs displaying uniform and compact morphology are ready to be harvested and expanded. D. Day 7 of failed neural induction. The cell population consists of flat non-neural cells with a heterogeneous morphology (indicated by red arrows) and compact NSCs/NPCs (indicated by blue arrows). To purify, it is required to pick up the compact NSCs/NPCs. E. P1 NSCs/NPCs at $24 \mathrm{~h}$ after the harvest and a passage of P0 NSCs/NPCs. F. P2 NSCs/NPCs show uniform morphology and grow in a honeycomb-like structure. Scale bars, $500 \mu \mathrm{m}$.

3. Precoat wells of a 6-well plate with $1 \mathrm{ml}$ Geltrex and incubate in a $37{ }^{\circ} \mathrm{C}, 5 \% \mathrm{CO}_{2}$ incubator for at least $1 \mathrm{~h}$.

4. When iPSCs culture reaches $70-80 \%$ confluence, detach iPSC colonies with collagenase IV 
solution. i.e., aspirate the iPSC medium, wash once with $5 \mathrm{ml}$ of PBS, and add $4 \mathrm{ml}$ of collagenase IV solution to the cell colonies.

5. Incubate the cells with collagenase IV solution in a $37^{\circ} \mathrm{C}, 5 \% \mathrm{CO}_{2}$ incubator for $30 \mathrm{~min}$. Note: If necessary, incubate 10-15 min longer but do not exceed 60 min to prevent the reduction of cell viability.

6. When edges of the colonies start to roll up, remove the collagenase IV solution and add $10 \mathrm{ml}$ iPSC medium to dilute and wash out collagenase IV.

7. Collect the detached colonies (after gentle rinsing with the iPSC medium using a $10 \mathrm{ml}$ pipette) into a 15-ml conical tube.

8. To remove MEF feeder cells, leave the tube containing cell suspension to stand for $10 \mathrm{~min}$. Then, aspirate the supernatant containing MEF without disturbing the IPSC pellet.

Note: iPSC colonies settle to the bottom by gravity, while MEF single cells do not.

9. Add a $6 \mathrm{ml}$ iPSC medium to generate cell suspension and follow the following Steps A9a-A9d to estimate a cell concentration.

a. Transfer a portion of the suspension $(1 \mathrm{ml})$ to a new $15-\mathrm{ml}$ conical tube.

b. Centrifuge the $15-\mathrm{ml}$ conical tube from $9 \mathrm{a}$ at $200 \times \mathrm{g}$ for $3 \mathrm{~min}$ and aspirate the supernatant.

c. To dissociate iPSC clusters into single cells, add $1 \mathrm{ml}$ of Accutase to the $15-\mathrm{ml}$ conical tube, followed by incubation for 5 min at $37^{\circ} \mathrm{C}$.

d. After pipetting the cells up and down, perform a cell count via your preferred method (e.g., Trypan blue exclusion method).

10. Aspirate the Geltrex coating buffer from Step A3 and add $2.5 \mathrm{ml}$ iPSC medium into a coated well of 6 -well plate.

11. Gently shake the conical tube containing the iPSCs suspension from Step A9, and plate the cells on the well at $2.5 \times 10^{5}-3 \times 10^{5}$ cells/well.

Note: iPSCs for neural differentiation should be prepared as clusters. Single iPSCs will not survive in the present protocol.

12. Add Y27632, a ROCK inhibitor, to the cell suspension in the well to a final concentration of $10 \mu \mathrm{M}$.

13. Rock the plate gently in a back-and-forth and side-to-side fashion to distribute the cells evenly. Note: Do not swirl the plate as this will cause uneven distribution of the iPSC clusters.

14. Place the plate and incubate the cells in a $37^{\circ} \mathrm{C}, 5 \% \mathrm{CO}_{2}$ incubator overnight.

B. The induction of iPSCs into neural stem/neural progenitor cells (NSCs/NPCs) (days 0-7)

1. On the next day (day 0 ) (Figure $2 \mathrm{~B}$ ), aspirate $\mathrm{iPSC}$ culture medium on the plate and add $2.5 \mathrm{ml}$ prewarmed Neural Induction Medium (NIM; Recipe 2) to the well of the 6-well plate. Then, return the plates to the incubator.

2. On day 2, change the medium with $2.5 \mathrm{ml}$ of prewarmed fresh NIM per well.

3. On day 4 , change the medium with $2.5 \mathrm{ml}$ of prewarmed fresh NIM per well.

4. On day 6 , change the medium with $2.5 \mathrm{ml}$ of prewarmed fresh NIM per well. 
5. On day 7, NSCs/NPCs (identified passage $0 ; \mathrm{P} 0$ ) are ready to be harvested and expanded (Figure 2C)

Note: The differentiated cells' morphology should be uniform and compact when NSCs/NPCs are successfully induced.

C. Expansion and cryopreservation of the NSCs/NPCs (day 7 )

1. Confirm that the morphology of resultant NSCs/NPCs is in a homogenous and compact shape. If you observe flat non-neural cells with a heterogeneous morphology (red arrows in Figure 2D), follow the following Steps C2a-C2g to purify the NSCs/NPCs from the non-neural cells.

a. Precoat a well of 6-well plates with Geltrex in the same manner as described above.

b. Aspirate the Geltrex from Step C1a and add $2.5 \mathrm{ml}$ prewarmed Neural Expansion Medium (NEM; see Recipes) containing $5 \mu \mathrm{M}$ of Y27632 into a coated well of 6-well plate.

c. Under an inverted microscope in the biological safety cabinet, pick the compact-shaped NSCs/NPCs (blue arrows in Figure 2D) by physically scratching them using a $20 \mu$ pipette tip.

d. Transfer the cells to the well containing NEM from Step C1b.

e. Repeat Steps C1c-C1d several times to collect NSCs/NPCs in the well.

Note: There is no minimum or maximum number of compact NSCs/NPCs, which can be transferred in one well; however, we recommend you collect cells as many as you can.

f. Rock the plate gently in a back-and-forth and side-to-side fashion to distribute the cells evenly and place the plate in a $37^{\circ} \mathrm{C}, 5 \% \mathrm{CO}_{2}$ incubator.

g. After overnight incubation, change to fresh NEM without Y27632 and return the plate to the incubator and incubate NSCs/NPCs for one more day.

h. The next day, confirm that the morphology of the cells is in a homogenous and compact shape.

2. Precoat wells of 6-well plates with Geltrex in the same manner as described above.

3. Aspirate medium from the well containing the NSCs/NPCs and gently add $2 \mathrm{ml}$ of PBS to rinse the cells.

4. Aspirate PBS; then, add $1 \mathrm{ml}$ Accutase to each well of the 6-well plates and incubate at $37^{\circ} \mathrm{C}$ for $5 \mathrm{~min}$ to detach the cells from the wells' surface.

5. After adding $1 \mathrm{ml}$ of PBS to the well of the 6-well plates, transfer the cell suspension to the 15$\mathrm{ml}$ conical tube followed by gentle pipetting of the cell suspension up and down 3 times to disrupt the cell clumps.

6. Centrifuge the tube at $300 \times g$ for $5 \mathrm{~min}$.

7. Aspirate the supernatant, resuspend the cells in $3 \mathrm{ml} \mathrm{PBS}$, and centrifuge the cells at $300 \times g$ for 5 min.

8. Aspirate the supernatant and resuspend the cells in $1 \mathrm{ml}$ prewarmed NEM.

9. Perform the cell count via your preferred method (e.g., Trypan blue exclusion method).

10. Dilute the cell suspension with prewarmed NEM to $2 \times 10^{5}-4 \times 10^{5} \mathrm{cells} / \mathrm{ml}$. 
11. Aspirate the Geltrex from Step $\mathrm{C} 2$ and plate $2.5 \mathrm{ml}$ of the cell suspension into the well $(2.5 \times$ $10^{5}-3 \times 10^{5}$ cells per well).

12. Add $\mathrm{Y} 27632$ to the cell suspension to a final concentration of $5 \mu \mathrm{M}$.

13. Rock the plate gently in a back-and-forth and side-to-side fashion to distribute the cells evenly. Note: Do not swirl the plate to prevent an uneven distribution of the cells.

14. The next day, aspirate the culture medium on the plate and add $2.5 \mathrm{ml}$ prewarmed fresh NEM without Y27632 to the well of the 6-well plate. The cells' culture medium has to be changed with $2.5 \mathrm{ml}$ of fresh prewarmed NEM every other day.

15. When NSCs/NPCs reach confluence, they can be further expanded by following Steps C2-C14 above, cryopreserved, differentiated to neurons or analyzed by immunofluorescence (Figure 3). Notes:

a. It is of great importance to passage the cells at a density of $0.5 \times 10^{5}-1 \times 10^{5} \mathrm{cells} / \mathrm{cm}^{2}(2 \times$ $10^{5}-4 \times 10^{5} \mathrm{cell} / \mathrm{s} / \mathrm{ml}$ ) since they easily lose their multipotency to differentiate to neuron/glial cells if they are grown at low density.

b. On passaging, we recommend you apply Y27632 to the medium to prevent cell death. Y27632 should be removed by replacing fresh medium the next day. 
A

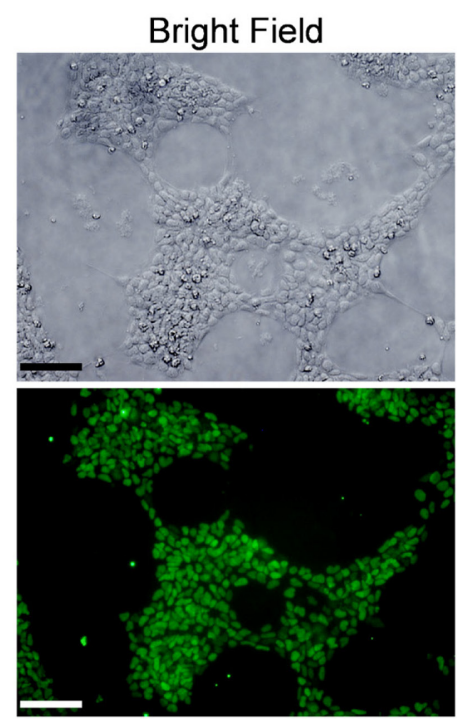

SOX1

B

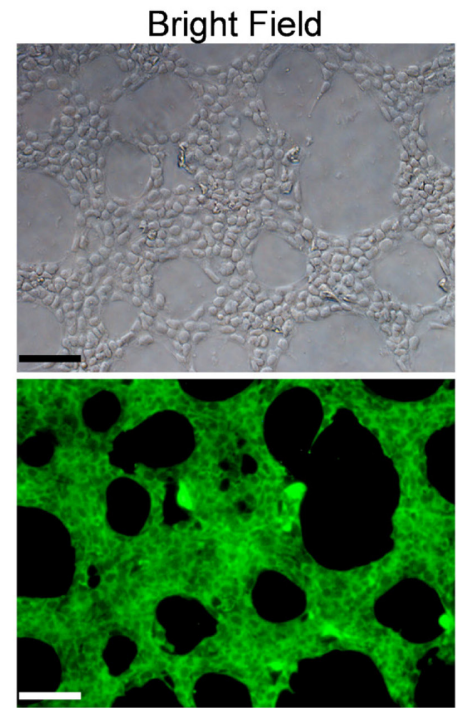

PSA-NCAM
Hoechst

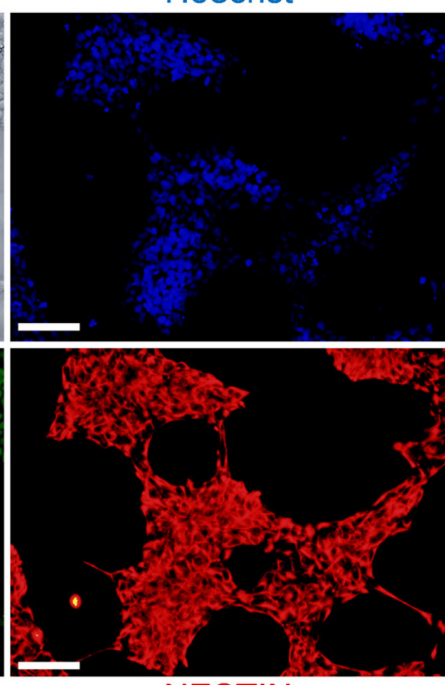

NESTIN

Hoechst

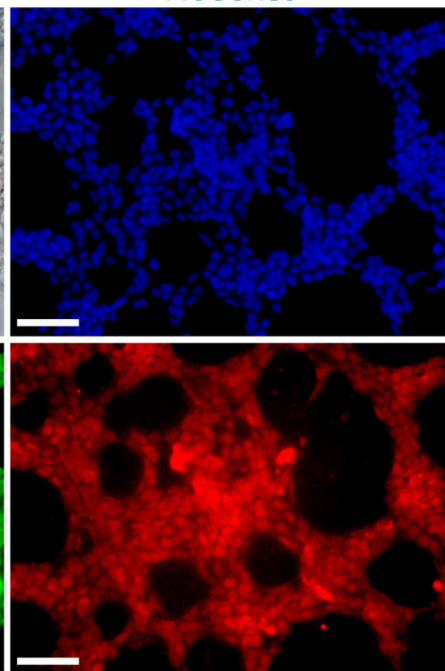

PAX6

Figure 3. Immunofluorescence staining of NSCs/NPCs with antibodies for neural stem cell markers. iPSC-derived NSCs/NPCs (P1) are immunostained with anti-SOX1 (green), antiNESTIN (red) (A), and anti-PSA-NCAM (green), anti-PAX6 (red) antibodies (B). Cell nuclear was identified by Hoechst staining (blue). Scale bars, $200 \mu \mathrm{m}$.

16. When NSCs/NPCs are ready to be cryopreserved, they can be detached by following Steps C3C9 above. After that, make cell suspension in NSC freezing medium (see Recipes) at $2 \times 10^{6}$ $4 \times 10^{6} \mathrm{cells} / \mathrm{ml}$, and dispense $1 \mathrm{ml}$ cell suspension into each cryovial and freeze cells at $-80^{\circ} \mathrm{C}$ overnight. If necessary, transfer the frozen vials to a liquid nitrogen storage tank after the vials have been frozen for at least $24 \mathrm{~h}$.

D. The differentiation and maturation of neurons from the NSCs/NPCs (neuronal day 0: N0-N56)

1. Before starting neuronal differentiation, precoat the surface of glass bottom dishes with $0.2 \mathrm{ml}$ 
polyethylenimine $(\mathrm{PEI})$ solution $\left(2.8 \times 10^{2} \mu \mathrm{M}\right.$; Recipe 5$)$ and incubate overnight at room temperature.

2. On the day of starting neuronal differentiation (day NO), wash the PEl-coated dishes with autoclaved water at least 5 times. Until use, keep the surface of the dishes covered with water after the final wash step.

Notes:

a. An extensive washout of the remaining PEI solution is necessary to reduce the cell toxicity by excess PEI.

b. The PEI-coated dishes can be stored at room temperature for up to 2 days.

3. Aspirate medium from the well of the 6-well plates containing NSCs/NPCs and gently add $2 \mathrm{ml}$ of PBS to rinse the cells.

4. Aspirate PBS. Then, add $1 \mathrm{ml}$ Accutase to each well of the 6-well plates and incubate at $37^{\circ} \mathrm{C}$ for $5 \mathrm{~min}$ to detach the cells from the wells' surface.

5. After adding $2 \mathrm{ml}$ of PBS to the well of the 6-well plates, transfer the cell suspension to the 15 $\mathrm{ml}$ conical tube followed by gentle pipetting of the cell suspension up and down 3 times to disrupt the cell clumps.

6. Centrifuge the tube at $300 \times g$ for $5 \mathrm{~min}$.

7. Aspirate the supernatant, resuspend the cells in $3 \mathrm{ml} \mathrm{PBS}$, and centrifuge the cells at $300 \times g$ for $5 \mathrm{~min}$.

8. Aspirate the supernatant and resuspend the cells in $1 \mathrm{ml}$ prewarmed Neuronal Medium (NM; Recipe 6).

9. Perform the cell count using your preferred method (e.g., Trypan blue exclusion method).

10. Dilute the cell suspension with prewarmed NM to $5 \times 10^{5}$ cells $/ \mathrm{ml}(0.2 \mathrm{ml}$ is needed for a well of the glass-bottom dish).

11. Aspirate the water on the PEl-coated dishes from step 2 and plate $0.2 \mathrm{ml}$ of the cell suspension on each dish $\left(1 \times 10^{5}\right.$ cells per dish: $\left.1 \times 10^{5} \mathrm{cells} / \mathrm{cm}^{2}\right)$.

12. Add Y27632 to the cell suspension to a final concentration of $5 \mu \mathrm{M}$.

13. Rock the dishes gently in a back-and-forth and side-to-side fashion to distribute the cells evenly. Note: Do not swirl the dishes to prevent an uneven distribution of the cells.

14. Place the cell dishes in a $37{ }^{\circ} \mathrm{C}, 5 \% \mathrm{CO}_{2}$ incubator overnight.

15. The next day, aspirate the culture medium and add $2 \mathrm{ml}$ prewarmed fresh NM without $\mathrm{Y} 27632$ to the dishes. Return the dishes to a $37^{\circ} \mathrm{C}, 5 \% \mathrm{CO}_{2}$ incubator.

16. Until day $\mathrm{N} 28$, half of the medium $(1 \mathrm{ml})$ for the cells must be changed carefully and gently with fresh prewarmed NM twice a week.

Note: The differentiated neurons are easy to be detached from the surface of the dishes by physical force such as moving the dish and removing medium; therefore, extreme care must be taken when performing medium change operation.

17. After 4 weeks of neuronal differentiation (day N28), change half of the medium with Neuronal Maturation Medium (NMM; Recipe 7). 
18. Continue to change half the medium with fresh NMM once or twice a week for further 4 weeks (at least until day N56) for neurons' maturation.

19. After 4 weeks of neuronal maturation (day N56 ), the neurons are ready to be analyzed by immunofluorescence (Figure 4) and functional assays such as FM1-43 exocytotic imaging.

A

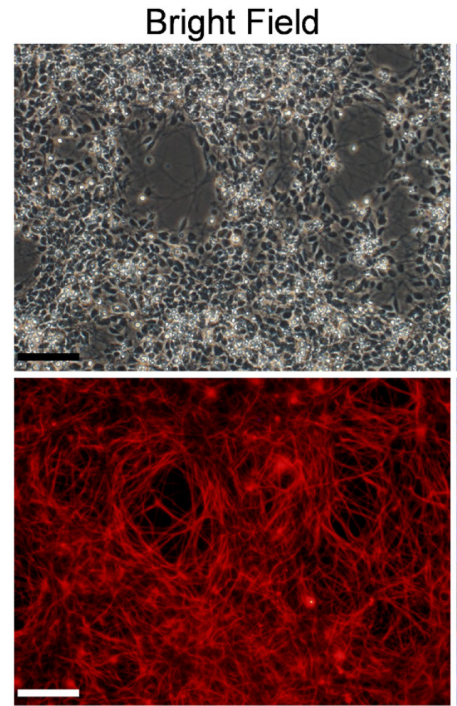

Tuj1

B

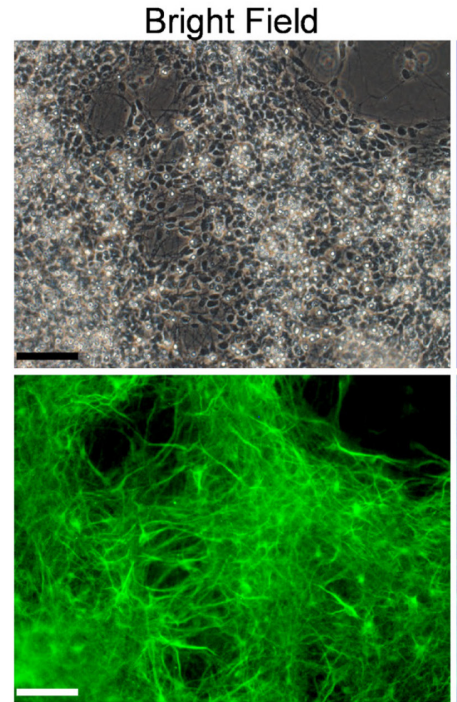

MAP2
Hoechst

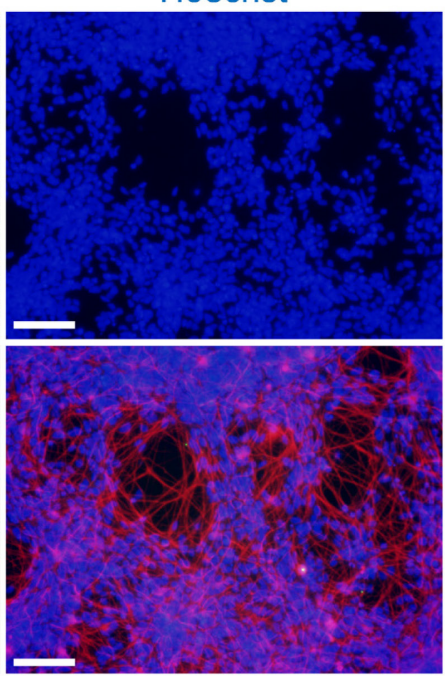

Merge

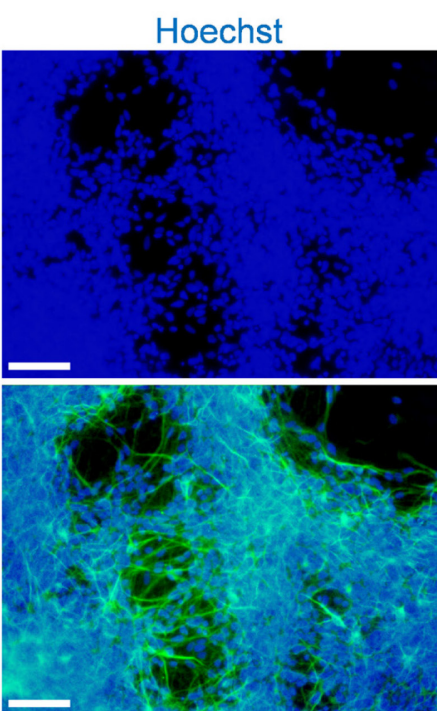

Merge

Figure 4. Immunofluorescence staining of neurons differentiated from NSCs/NPCs. iPSCderived neurons are immunostained with anti-Tuj1 (red) (A), and anti-MAP2 (green) antibodies (B). Cell nuclear was also stained with Hoechst (blue). Scale bars, $200 \mu \mathrm{m}$.

E. Immunofluorescence (Video 1)

1. Aspirate the culture medium without dislodging the cells.

Note: The differentiated cells, especially neurons, are easily detached from the dishes' surface by physical force; therefore, extreme care must be taken when adding and removing buffers. 
2. Fix the cells with $4 \%$ Paraformaldehyde Phosphate Buffer Solution at room temperature for 15 min.

3. After aspirating the paraformaldehyde solution, rinse the cells 3 times with PBS.

4. Permeabilize the cells with $0.1 \%$ Triton $\mathrm{X}-100$ in $\mathrm{PBS}$ at room temperature for $10 \mathrm{~min}$.

5. After aspirating the permeabilization buffer, block the cells with 5\% BSA in PBS at room temperature for $60 \mathrm{~min}$.

6. After aspirating the blocking buffer, immunostain the cells with primary antibodies diluted in blocking buffer overnight at $4{ }^{\circ} \mathrm{C}$. Anti-SOX1 (rabbit; dilution 1:100), anti-NESTIN (mouse IgG; 1:500), anti-PSA-NCAM (mouse IgM; 1:400) and anti-PAX6 (rabbit; dilution 1:1,000) antibodies are used for NSCs/NPCs, and anti-Tuj1 (mouse IgG; 1:500), anti-MAP2 (rabbit; 1:500) antibodies for neurons, respectively.

7. After aspirating the primary antibodies, wash the cells with PBS 3 times to rinse off the nonbinding primary antibodies.

8. Incubate the cells with relevant fluorescence-labeled secondary antibodies diluted in PBS at room temperature for $60 \mathrm{~min}$ in the dark. Alexa 488-conjugated anti-rabbit IgG $(1: 1,000)$, Alexa 488-conjugated goat anti-mouse IgM (1:1,000), Alexa 594-conjugated donkey anti-rabbit $\lg G(1: 1,000)$, and Alexa 594-conjugated anti-mouse IgG $(1: 1,000)$ are used.

9. After aspirating the secondary antibodies, wash the cells with PBS 3 times to rinse off the nonbinding secondary antibodies, and in the last wash, counterstain the nuclei with Hoechst 33258 $(1 \mu \mathrm{g} / \mathrm{ml})$.

10. Analyze the cells on a fluorescence microscope.

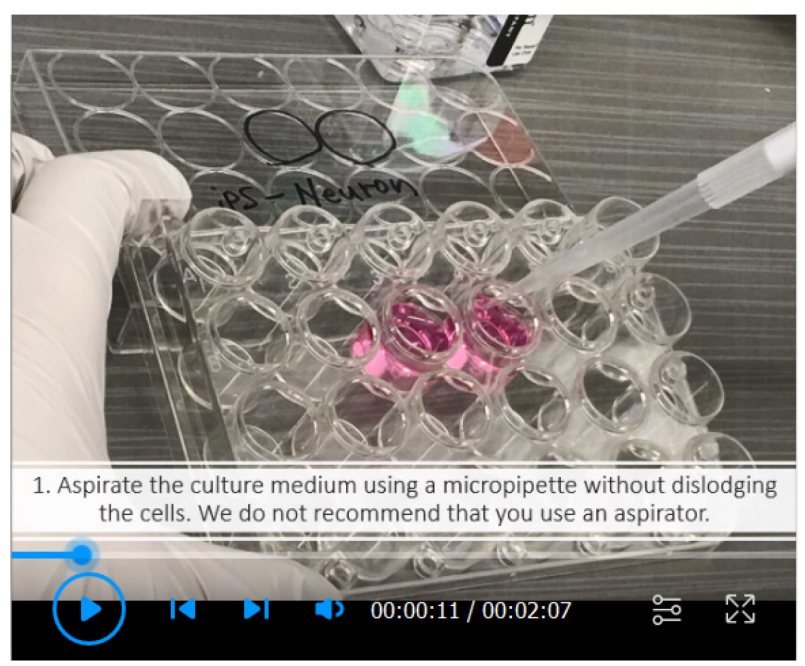

Video 1. Immunofluorescence Tutorial. In this tutorial, we cover the basics of immunostaining cells with primary and fluorescence-labeled secondary antibodies.

F. Functional analysis of mature neurons with FM1-43 fluorescent dye.

1. Remove the neurons' culture medium maintained on the glass-bottom dish using a 1,000 $\mu \mathrm{l}-$ micropipette without dislodging the cells. 
Note: It is important to use the micropipette for removing the medium. Do not use an aspirator as it can damage the neurons during the process.

2. Gently wash the cells once with $200 \mu$ prewarmed Artificial Cerebrospinal Fluid (ACSF; Recipe 8) using a 1,000 $\mu$-micropipette without dislodging the cells.

3. Incubate the cells with $200 \mu \mathrm{l} \mathrm{ACSF}$ containing $5 \mu \mathrm{M}$ of $\mathrm{FM} 1-43$ for an hour in a $37{ }^{\circ} \mathrm{C}, 5 \% \mathrm{CO}_{2}$ incubator.

4. Remove the FM1-43 solution using 1,000 $\mu$ l-micropipette without disturbing the cells.

5. Gently wash the cells 3 times with $200 \mu$ prewarmed ACSF using 1,000 $\mu$-micropipette without dislodging the cells, as described in step2.

6. Immerse the cells with $2 \mathrm{ml}$ fresh ACSF without FM1-43.

7. Analyze the FM1-43 intensity of the cells on a fluorescence microscope (Leica).

Note: FM1-43 should be excited using a $488 \mathrm{~nm}$ (wavelength) blue light laser and its emission $(520 \mathrm{~nm})$ is acquired every second as a series of images through a $\times 40$ objective lens. For analysis, the quenching of FM1-43 caused by the treatment with high potassium (KCl) solution at $50 \mathrm{mM}$ can be considered an exocytotic activity. 
A

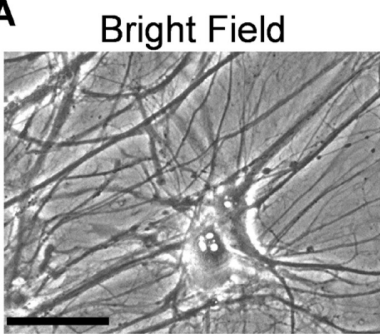

FM1-43

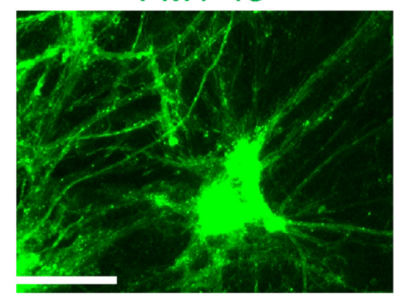

Before the stimulation

B

Bright Field
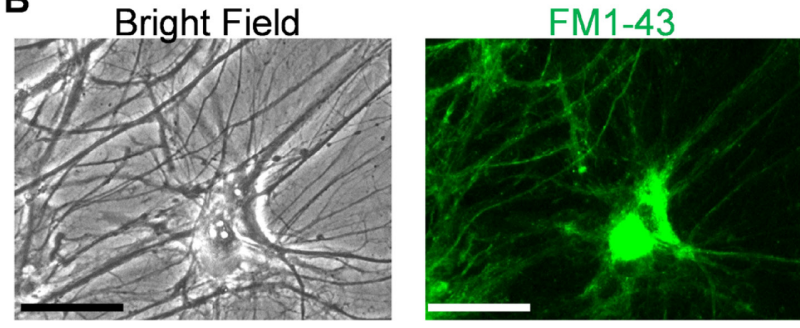

$60 \mathrm{sec}$ after the stimulation

C

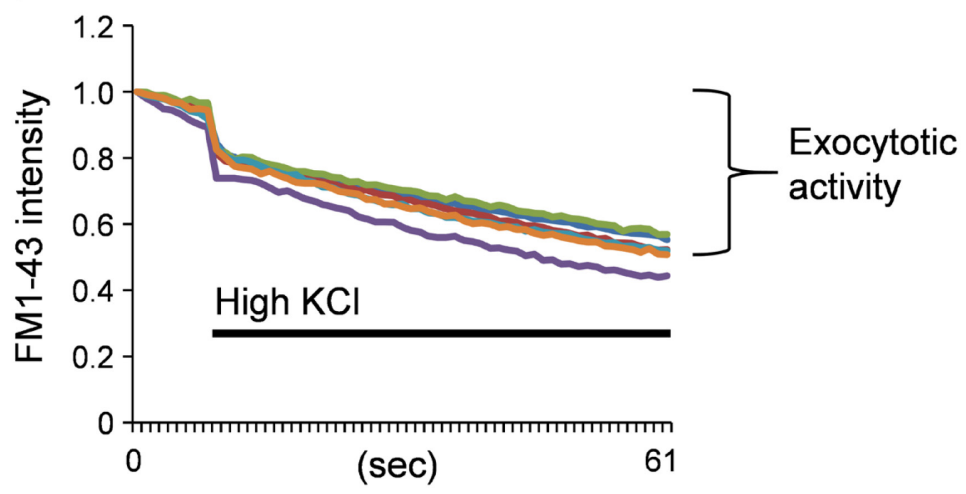

Figure 5. Functional analysis of human neurons with FM1-43 fluorescent dye. Mature neurons were used for functional analysis with FM1-43 imaging, a powerful tool to determine exocytotic activity. A. Bright and fluorescent images of neurons loaded with FM1-43 dye before the stimulation to induce cell depolarization. B. $60 \mathrm{~s}$ after the stimulation. Scale bars, $25 \mu \mathrm{m}$. C. The time-course of the intensity of FM1-43 fluorescence before and after the stimulation. The reduction of FM1-43 intensity can be recognized as an exocytosis. A high $\mathrm{KCl}$ solution (final 50 $\mathrm{mM}$ ) was added to evoke the cell depolarization.

\section{Data analysis}

The change of intracellular FM1-43 fluorescence was determined during the before and after depolarization stimulation with $50 \mathrm{mM} \mathrm{HK}$. The emission of FM1-43 was captured every one second and stored as laminated images using the LAS X imaging system. Detailed analyses of iPSCinduced neurons by this protocol can be found in our recent publication (Supplemental information in Kajihara et al., 2020). 


\section{$\underline{\text { Recipes }}$}

1. iPSC medium

$\begin{array}{ll}\text { DMEM/F12 } & 400 \mathrm{ml} \\ \text { NON-ESSENTIAL AMINO ACID (X100) } & 5 \mathrm{ml} \\ 200 \mathrm{mM} \text { L-GLUTAMINE } & 5 \mathrm{ml} \\ \text { KnockOut Serum Replacement } & 100 \mathrm{ml} \\ 0.1 \mathrm{M} \text { 2-MERCAPTOETHANOL/PBS } & 500 \mu \mathrm{l} \\ 10 \mu \mathrm{g} / \mathrm{ml} \text { bFGF in 0.1\% BSA/PBS } & 200 \mu \mathrm{l}\end{array}$

Total: $510.7 \mathrm{ml}$

The medium can be stored in the dark at $2-8^{\circ} \mathrm{C}$ for up to 2 weeks.

2. Neural Induction Medium

Neurobasal Medium $49 \mathrm{ml}$

Neural Induction Supplement $(\times 50)$

Total: $50 \mathrm{ml}$

The medium can be stored in the dark at $2-8^{\circ} \mathrm{C}$ for up to 2 weeks.

Note: Before using, warm the medium in a water bath at $37^{\circ} \mathrm{C}$ for 5-10 min. However, do not warm the medium in a $37^{\circ} \mathrm{C}$ water bath for more than 10 min since the components in the medium may deteriorate.

3. Neural Expansion Medium

Neurobasal Medium

$24.5 \mathrm{ml}$

Advanced DMEMF12

$24.5 \mathrm{ml}$

Neural Induction Supplement $(\times 50)$

$1 \mathrm{ml}$

Total: $50 \mathrm{ml}$

The medium can be stored in the dark at $2-8^{\circ} \mathrm{C}$ for up to 2 weeks.

Note: Before using, warm the medium in a water bath at $37^{\circ} \mathrm{C}$ for $5-10 \mathrm{~min}$. However, do not warm the medium in a $37{ }^{\circ} \mathrm{C}$ water bath for more than 10 min to avoid damage of the components in the medium.

4. NSC freezing medium

Neural Expansion Medium $\quad 9 \mathrm{ml}$

DMSO $1 \mathrm{ml}$

Total: $10 \mathrm{ml}$

5. Polyethylenimine (PEI) solution

Polyethylenimine $1 \mathrm{ml}$

$\mathrm{H}_{2} \mathrm{O}$, Milli-Q-filtered $499 \mathrm{ml}$

Total: $500 \mathrm{ml}$

6. Neuronal Medium

Neurobasal Medium $49 \mathrm{ml}$

B27 Supplement $(\times 50) \quad 1 \mathrm{ml}$ 
Total: $50 \mathrm{ml}$

The medium can be stored in the dark at $2-8^{\circ} \mathrm{C}$ for up to 2 weeks.

7. Neuronal Maturation Medium

$\begin{array}{ll}\text { DMEM } & 40 \mathrm{ml} \\ \text { KnockOut Serum Replacement } & 10 \mathrm{ml}\end{array}$

Total: $50 \mathrm{ml}$

The medium can be stored in the dark at $2-8^{\circ} \mathrm{C}$ for up to 2 weeks.

8. Artificial Cerebrospinal Fluid

$\begin{array}{ll}\mathrm{NaCl} & 7.3 \mathrm{~g} \\ \mathrm{KCl} & 0.1864 \mathrm{~g} \\ \mathrm{CaCl}_{2} \cdot 2 \mathrm{H}_{2} \mathrm{O} & 0.294 \mathrm{~g} \\ \mathrm{MgCl}_{2} \cdot 6 \mathrm{H}_{2} \mathrm{O} & 0.2024 \mathrm{~g} \\ \mathrm{NaH}_{2} \mathrm{PO}_{4} \cdot 2 \mathrm{H}_{2} \mathrm{O} & 0.195 \mathrm{~g} \\ \mathrm{NaHCO}_{3} & 2.1 \mathrm{~g} \\ \mathrm{Glucose} & 4.5 \mathrm{~g} \\ \mathrm{H}_{2} \mathrm{O}, \text { Milli-Q-filtered } & \text { Up to } 1,000 \mathrm{ml}\end{array}$

\section{Acknowledgments}

This study was supported, in part, by grants from Japan Society for the Promotion of Science KAKENHI grant number 18K15369 and 20K06857. We are grateful to Dr. Haruki Odaka for his advice on neural induction. This protocol was derived from our previous work (Kajihara et al., 2020).

\section{Competing interests}

The authors declare that they have no conflict of interest.

\section{Ethics}

The present protocol using human iPSC was performed in accordance with approval from the Ethics

Committee for Gene Analysis and Genome Research of Kumamoto University. Informed consent was obtained from all subjects.

\section{References}

1. Chambers, S. M., Fasano, C. A., Papapetrou, E. P., Tomishima, M., Sadelain, M. and Studer, L. (2009). Highly efficient neural conversion of human ES and iPS cells by dual inhibition of SMAD signaling. Nat Biotechnol 27(3): 275-280.

2. Dimos, J. T., Rodolfa, K. T., Niakan, K. K., Weisenthal, L. M., Mitsumoto, H., Chung, W., Croft, 
G. F., Saphier, G., Leibel, R., Goland, R., Wichterle, H., Henderson, C. E. and Eggan, K. (2008). Induced pluripotent stem cells generated from patients with ALS can be differentiated into motor neurons. Science 321(5893): 1218-1221.

3. Dunn, N. R., Vincent, S. D., Oxburgh, L., Robertson, E. J. and Bikoff, E. K. (2004). Combinatorial activities of Smad2 and Smad3 regulate mesoderm formation and patterning in the mouse embryo. Development 131(8): 1717-1728.

4. Era, T., Izumi, N., Hayashi, M., Tada, S., Nishikawa, S. and Nishikawa, S. I. (2008). Multiple mesoderm subsets give rise to endothelial cells, whereas hematopoietic cells are differentiated only from a restricted subset in embryonic stem cell differentiation culture. Stem Cells 26(2): 401-411.

5. Falk, A., Koch, P., Kesavan, J., Takashima, Y., Ladewig, J., Alexander, M., Wiskow, O., Tailor, J., Trotter, M., Pollard, S., Smith, A. and Brustle, O. (2012). Capture of neuroepithelial-like stem cells from pluripotent stem cells provides a versatile system for in vitro production of human neurons. PLoS One 7(1): e29597.

6. Inoue, H., Nagata, N., Kurokawa, H. and Yamanaka, S. (2014). iPS cells: a game changer for future medicine. EMBO J 33(5): 409-417.

7. Kajihara, R., Numakawa, T., Odaka, H., Yaginuma, Y., Fusaki, N., Okumiya, T., Furuya, H., Inui, S. and Era, T. (2020). Novel Drug Candidates Improve Ganglioside Accumulation and Neural Dysfunction in GM1 Gangliosidosis Models with Autophagy Activation. Stem Cell Reports 14(5): 909-923.

8. Kawasaki, H., Mizuseki, K., Nishikawa, S., Kaneko, S., Kuwana, Y., Nakanishi, S., Nishikawa, S.-I. and Sasai, Y. (2000). Induction of midbrain dopaminergic neurons from ES cells by stromal cell-derived inducing activity. Neuron 28(1): 31-40.

9. Kido, J., Nakamura, K. and Era, T. (2020). Role of induced pluripotent stem cells in lysosomal storage diseases. Mol Cell Neurosci 103540.

10. Lee, H., Shamy, G. A., Elkabetz, Y., Schofield, C. M., Harrsion, N. L., Panagiotakos, G., Socci, N. D., Tabar, V. and Studer, L. (2007). Directed differentiation and transplantation of human embryonic stem cell-derived motoneurons. Stem Cells 25(8): 1931-1939.

11. Li, W., Sun, W., Zhang, Y., Wei, W., Ambasudhan, R., Xia, P., Talantova, M., Lin, T., Kim, J. and Wang, X. (2011). Rapid induction and long-term self-renewal of primitive neural precursors from human embryonic stem cells by small molecule inhibitors. Proc Natl Acad Sci 108(20): 82998304.

12. Marin Navarro, A., Susanto, E., Falk, A. and Wilhelm, M. (2018). Modeling cancer using patientderived induced pluripotent stem cells to understand development of childhood malignancies. Cell Death Discov 4: 7.

13. Matsushita, K., Numakawa, T., Odaka, H., Kajihara, R., Soga, M., Ozasa, S., Nakamura, K., Mizuta, H. and Era, T. (2019). Presynaptic Dysfunction in Neurons Derived from Tay-Sachs iPSCs. Neuroscience 414: 128-140.

14. Morizane, A., Doi, D., Kikuchi, T., Nishimura, K. and Takahashi, J. (2011). Small-molecule 
inhibitors of bone morphogenic protein and activin/nodal signals promote highly efficient neural induction from human pluripotent stem cells. J Neurosci Res 89(2): 117-126.

15. Nemati, S., Hatami, M., Kiani, S., Hemmesi, K., Gourabi, H., Masoudi, N., Alaei, S. and Baharvand, H. (2011). Long-term self-renewable feeder-free human induced pluripotent stem cell-derived neural progenitors. Stem Cells Dev 20(3): 503-514.

16. Nishikawa, S.-I., Jakt, L. M. and Era, T. (2007). Embryonic stem-cell culture as a tool for developmental cell biology. Nat Rev Mol Cell Biol 8(6): 502-507.

17. Raab, S., Klingenstein, M., Liebau, S. and Linta, L. (2014). A Comparative View on Human Somatic Cell Sources for iPSC Generation. Stem Cells Int 2014: 768391.

18. Saha, B., Borgohain, M., Dey, C. and Thummer, R. (2018). iPS cell generation: current and future challenges. Ann Stem Cell Res Ther 1(2): 1007.

19. Shi, Y., Kirwan, P. and Livesey, F. J. (2012). Directed differentiation of human pluripotent stem cells to cerebral cortex neurons and neural networks. Nat Protoc 7(10): 1836-1846.

20. Soga, M., Ishitsuka, Y., Hamasaki, M., Yoneda, K., Furuya, H., Matsuo, M., Ihn, H., Fusaki, N., Nakamura, K. and Nakagata, N. (2015). HPGCD Outperforms HPBCD as a Potential Treatment for Niemann-Pick Disease Type C During Disease Modeling with iPS Cells. Stem Cells 33(4): 1075-1088.

21. Tada, S., Era, T., Furusawa, C., Sakurai, H., Nishikawa, S., Kinoshita, M., Nakao, K., Chiba, T. and Nishikawa, S.-I. (2005). Characterization of mesendoderm: a diverging point of the definitive endoderm and mesoderm in embryonic stem cell differentiation culture. Development 132(19): 4363-4374.

22. Takahashi, K., Tanabe, K., Ohnuki, M., Narita, M., Ichisaka, T., Tomoda, K. and Yamanaka, S. (2007). Induction of pluripotent stem cells from adult human fibroblasts by defined factors. Cell 131(5): 861-872.

23. Wang, L. and Chen, Y.-G. (2016). Signaling control of differentiation of embryonic stem cells toward mesendoderm. J Mol Biol 428(7): 1409-1422.

24. Yan, Y., Shin, S., Jha, B. S., Liu, Q., Sheng, J., Li, F., Zhan, M., Davis, J., Bharti, K., Zeng, X., Rao, M., Malik, N. and Vemuri, M. C. (2013). Efficient and rapid derivation of primitive neural stem cells and generation of brain subtype neurons from human pluripotent stem cells. Stem Cells Trans/ Med 2(11): 862-870.

25. Yu, J., Vodyanik, M. A., Smuga-Otto, K., Antosiewicz-Bourget, J., Frane, J. L., Tian, S., Nie, J., Jonsdottir, G. A., Ruotti, V. and Stewart, R. (2007). Induced pluripotent stem cell lines derived from human somatic cells. Science 318(5858): 1917-1920. 